\title{
La calidad del sistema universitario en China: la Liga C9 y la clasificación de Shanghái
}

\section{The quality of the university system in China: The C9 League and the Shanghai Ranking}

NURIA RUIZ MORILLAS*

Resumen: El sistema universitario chino ha experimentado un crecimiento muy importante en los últimos años. El gobierno ha destinado recursos para potenciar la investigación y aumentar la presencia de las universidades chinas en el mapa universitario mundial. Nueve de éstas constituyen la Liga C9. En este artículo se analiza la evolución de las posiciones en la clasificación de Shanghái de las universidades de la Liga C9 y se comparan los resultados con otras dos clasificaciones de carácter internacional, el QS World University Rankings y el Times Higher Education.

Palabras clave: calidad de la educación; universidades; educación pública; ranking académico.

Abstract: The Chinese university system has experienced significant growth in recent years. The government has allocated resources to boost research and increase the presence of Chinese universities on the world university map. Nine of these universities constitute the

Recepción: 9 de diciembre de 2016. / Aceptación: 14 de junio de 2018.

* Universidad Rovira i Virgili, nuria.ruizm@urv.cat 
C9 League. In this paper, we analyze the evolution of positions in the Shanghai ranking of C9 League universities. We also compare these results with two other international classifications, the QS World University Rankings and Times Higher Education.

Keywords: educational quality; universities; public education; academic ranking.

\section{Introducción}

Deng Xiaoping consideraba la educación como una contribución fundamental a la modernización del país. Desde finales de la década de 1980, las reformas al sistema educativo chino han sido continuas, y se incrementaron en los noventa. En el ámbito de las universidades, era necesario enfocarlas hacia la nueva realidad que experimentaban. Se quería impulsar la investigación para que contribuyera al desarrollo económico, ayudara a superar la pobreza y convirtiera a China en potencia global. Puesto que el gobierno tiene el poder de aprobar nuevos títulos, activar programas de doctorado y promocionar profesores, las políticas educativas determinan la calidad de las instituciones de educación superior (Xiao y Dyson, 1999, p. 345). En este sentido, a finales de los años noventa, el gobierno de $\mathrm{Hu}$ Jintao y Wen Jiabao consideró que el avance de la ciencia y la tecnología era prioritario para situar a China en un contexto internacional y se iniciaron distintos proyectos para conseguirlo.

Uno de los planes decisivos para promover el crecimiento de las universidades chinas, anunciado en 1995, fue el Proyecto 211 . Su nombre indicaba que cien de estas instituciones tenían que alcanzar un nivel adecuado de investigación e innovación para ser consideradas universidades del siglo 21 (XXI) a escala internacional. Con este objetivo, el gobierno seleccionó las mejores universidades e invirtió en ellas para potenciar el desarrollo de disciplinas técnicas y científicas, especialmente en el ámbito de la investigación (Zhang, Zhao y Lei, 2012, p. 264). Finalmente, 117 instituciones de educación superior (6\% del total) se incorporaron al proyecto y aceptaron la responsabilidad de formar a cuatro quintas partes de los estu- 
diantes de doctorado, dos tercios de posgrado, la mitad de los extranjeros y un tercio de los graduados. Es decir, una pequeña parte de las universidades chinas se encargaría de preparar a la mayor parte de los investigadores nacionales y extranjeros. Para ello, estas universidades recibían $70 \%$ del fondo público de investigación.

Otro plan importante fue el Proyecto 985, implementado en mayo de 1998 (de ahí su nombre) bajo el mandato del presidente Jiang Zemin. El objetivo era crear un grupo de universidades de élite y complementar el Proyecto 211 al invertir en ellas recursos extra para colocarlas en el mapa mundial de las universidades. Inicialmente, se concentraron en nueve universidades, y en 2004 el número se amplió a 39 (Zhao y Zhu, 2010, p. 123). La financiación combinaba aportaciones del gobierno central y de los gobiernos provinciales para construir nuevos centros de investigación, mejorar los resultados en investigación, participar en congresos internacionales, intensificar las colaboraciones y atraer a investigadores extranjeros altamente calificados. Las primeras nueve universidades beneficiadas (Pekín, Tsinghua, Fudan, Shanghái Jiao Tong, Nanjing, Ciencia y Tecnología de China, Zhejiang, Xi'an Jiaotong e Instituto de Tecnología de Harbin) formaron la Liga C9, una asociación que pretendía compartir recursos, personal y resultados para mejorar su productividad científica.

Así, la política de financiamiento de la educación superior se concentró en fortalecer unas cuantas universidades seleccionadas por el gobierno entre las dos mil que hay, aproximadamente, en el país, lo que provocó cierto malestar en las que no fueron escogidas y reciben menos presupuesto. También entre las universidades mejor situadas hay diferencias, porque a menudo las de Pekín y Tsinghua reciben la mayor parte de las subvenciones. A pesar de esto, con la ayuda de estos proyectos y otras iniciativas el gobierno chino ha conseguido que las instituciones de élite experimenten un progreso significativo, especialmente en términos de producción científica.

Entre 1995 y 2005, el número anual de artículos científicos producidos en China pasó de 9061 a 41596. En 2008, la cantidad aumentó a 112000 . El número de textos publicados por investigadores chinos en las cinco revistas más valoradas del 
mundo, Nature, Science, Cell, Lancet y Nerw England Journal of Medicine, se triplicó y alcanzó la cifra de 21 artículos anuales (Yang y Welch, 2012, p. 662). Así pues, las estrategias aplicadas por los gobiernos de Deng Xiaoping, Hu Jintao y Jiang Zeming han conseguido promocionar a las universidades chinas en el mapa mundial de la educación superior.

Una de las formas de visibilizar la calidad relativa de las universidades es a través de un ranking que aplique una rigurosa metodología científica de tipo bibliométrico e incluya criterios objetivos, medibles y reproducibles. La clasificación de Shanghái es uno de ellos.

Este trabajo analiza el progreso de las universidades chinas, concretamente de las nueve instituciones de élite de la Liga C9, a partir de los datos publicados en la clasificación de Shanghái, y valora las estrategias llevadas a cabo para mejorar las posiciones en el ranking. Además, dado que las universidades no suelen fijarse en un solo ranking, se analizan brevemente las posiciones de las que componen la Liga C9 en el QS World University Rankings y en el Times Higher Education.

\section{Las universidades chinas: la Liga C9}

En el marco del Proyecto 985, se seleccionaron en una primera fase (año 2009) nueve universidades repartidas por todo el territorio a las que se les asignó un presupuesto especial por un periodo de tres años:

- En Pekín: universidades de Pekín y Tsinghua.

- En los alrededores del delta del río Yangtze, al este de China: universidades de Fudan (Shanghái), Shanghái Jiao Tong (Shanghái), Nanjing (Nanjing, provincia de Jiangsu), Ciencia y Tecnología de China (Hefei, provincia de Anhui) y Zhejiang (Hangzhou, provincia de Zhejiang).

- En Xian (provincia de Shaanxi), al oeste de China: Universidad de Xi'an Jiaotong.

- En Harbin (provincia de Heilongjiang), al nordeste de China: Instituto de Tecnología de Harbin. 
De las nueve universidades, cuatro están en Pekín y en Shanghái, dos en capitales de provincias ricas (Hangzhou y Nanjing) $\mathrm{y}$ tres en capitales de provincias pobres (Xian, Hefei y Harbin). Además, no se escogieron sólo las situadas en provincias donde el nivel medio de educación es alto (en Pekín, 37.5\% de la población tiene estudios superiores, y en Shanghái, 27.1\%), sino también algunas de zonas menos favorecidas, donde el nivel educativo es inferior (en Shaanxi, sólo 10.7\% tiene estudios superiores, y en Heilongjiang, 10.1\%) (National Bureau of Statistics of China, 2013).

En 2009, estas universidades constituyeron la Liga C9, nombre inspirado en la Ivy League de Estados Unidos. Sin embargo, las instituciones que las componen no son comparables. En el caso norteamericano, las ocho universidades de la Ivy League (Brown, Columbia, Cornell, Darmouth College, Harvard, Pensilvania, Yale y Princeton) son privadas, de excelencia, y se unieron en el año 1954 para constituir una liga deportiva competitiva. En el caso chino, las nueve universidades son públicas y pretenden optimizar recursos para aumentar su excelencia académica y de investigación.

Las universidades de la Liga C9 fueron escogidas por el gobierno chino con la intención de que formaran un grupo y trabajaran juntas. El 12 de octubre de 2009 firmaron acuerdos de cooperación que favorecían los programas de intercambio de estudiantes, potenciaban la formación de posgraduados y establecían un sistema de créditos flexible entre los miembros de la Liga C9 (Yang y Welch, 2012, p. 647). Posteriormente, en 2013, las nueve universidades, junto con la Liga de Universidades Europeas de Investigación, el grupo Ocho Universidades de Australia y la Asociación de Universidades Americanas, firmaron una declaración que definía las características de las universidades de investigación contemporáneas. Según este comunicado, una universidad de investigación debe producir y difundir conocimiento a través de la investigación, la docencia y los servicios, sin restricciones, dentro de una cultura basada en la investigación abierta. En palabras del profesor Jianguo Hou, coordinador de la Liga C9 y presidente de la Universidad de Ciencia y Tecnología de China: "Esta declaración articula las características clave de las universidades de investigación que les 
permiten cumplir sus misiones de investigación y educación. Esboza los valores compartidos que sustentan la colaboración institucional transnacional efectiva" (Heron, 2013). Por lo tanto, se intuye una buena disposición para colaborar entre las escuelas chinas y las del resto del mundo.

Para darse a conocer, las nueve universidades disponen de páginas web muy completas con información pública en chino y en inglés. En este sentido, las nuevas tecnologías desempeñan un papel clave en la visibilidad de las universidades chinas, al favorecer la internacionalización y la competencia entre ellas para atraer talento académico. La divulgación de la misión, la oferta académica, los éxitos docentes, los investigadores y el funcionamiento de los órganos de gobierno fomentan también la competencia en el marco internacional (Flórez-Parra, López-Pérez y López-Hernández, 2014, p. 174). Así, la estética y los contenidos son semejantes a los de las páginas web de las mejores universidades anglosajonas, aunque ello suponga cierta desconexión con el territorio y la población local.

\section{La clasificación de Shanghái}

La clasificación de Shanghái (Academic Ranking of World Universities, ARWU) es elaborada anualmente por el Institute of Higher Education de la Universidad de Shanghái Jiao Tong. El propósito inicial de este ranking, creado en 2003, era ubicar la posición global de las mejores universidades de China. Se pretendía disponer de una herramienta que explicara las diferencias de calidad entre las instituciones chinas y las del mundo, para reducirlas y mejorar posiciones dentro del ranking. Gracias al buen diseño del método y a una elección adecuada de los elementos de cálculo, el interés se ha extendido a otros países y la clasificación de Shanghái se tiene en cuenta prácticamente en todo el mundo (Docampo y Cram, 2014, p. 1364).

La clasificación de Shanghái considera para su estudio aquellas universidades en que ha estudiado algún premio Nobel o receptor de premios internacionales relevantes, o que cuenta con investigadores citados frecuentemente o con artículos 
publicados en Nature o Science. También incluye universidades que publican artículos en revistas indexadas en el Science Citation Index-Expanded (SCIE) y en el Social Science Citation Index (SSCI). En total se contemplan más de 1200, y se registran ordenadas las 500 mejores.

El proceso de evaluación se lleva a cabo a través de indicadores. Para cada indicador se asignan 100 puntos a la institución con valoración más alta, y a las otras escuelas se les adjudica un porcentaje de esta puntuación.

Los indicadores son:

- Calidad de la docencia (número de antiguos alumnos que han recibido premios Nobel u otros premios relevantes): $10 \%$.

- Calidad del profesorado (número de académicos que han recibido premios relevantes y número de investigadores altamente citados e identificados en la base de datos Thomson Scientific): $40 \%$.

- Producción investigadora (número de artículos publicados en Nature y Science en los últimos cinco años y número de artículos publicados en otras revistas indexadas en Thomson Scientific en el último año): 40\%.

- Rendimiento académico per cápita (cálculo que consiste en dividir la puntuación de los indicadores anteriores entre el número de personal académico de tiempo completo): $10 \%$.

En el ranking, aparecen ordenadas las 100 primeras universidades de todo el mundo según los criterios anteriores. El resto se muestran en grupos de 50 (hasta la 201), y después en grupos de 100 (Billaut, Bouyssou y Vincke, 2010, p. 239).

Ante el éxito mundial de la clasificación de Shanghái y para satisfacer las necesidades de los usuarios, en 2007 se ampliaron las estadísticas y se publicaron los nombres de las 200 mejores universidades divididas en cinco grandes áreas de conocimiento (ARWU-Field: Ciencias Naturales y Matemáticas, Ingeniería/Tecnología y Ciencias de la Computación, Ciencias de la Vida y de Agricultura, Medicina Clínica y Farmacia, y Ciencias Sociales). En 2009 también se divulgaron las 200 uni- 
versidades clasificadas en cinco materias (ARWU-Subject: Matemáticas, Física, Química, Ciencias de la Computación y Economía/Negocios).

Como se observa a partir de la descripción de los indicadores, la clasificación de Shanghái está fuertemente relacionada con la producción investigadora de las universidades. Incluso los indicadores docentes (calidad de la docencia y el profesorado) tienen que ver con la investigación porque lo que se valora es el número de antiguos estudiantes o profesores con premios Nobel o similares, es decir, que tengan actividad importante en investigación. La clasificación de Shanghái da por sentado, pues, que disponer de profesores con premios relevantes internacionalmente, y muy citados en revistas de alto impacto científico, es un factor determinante para la calidad docente. También asume que los antiguos estudiantes distinguidos en investigación lo han sido gracias a la docencia de calidad recibida durante sus estudios universitarios. Aunque esta asunción es cuestionable porque puede haber otros criterios que reflejen la calidad docente (asignaturas impartidas, relación número de estudiantes graduados/estudiantes matriculados), hay que reconocer que sólo los criterios que no provienen de las instituciones son objetivos y rigurosos, porque los que proporcionan las universidades son manipulables y, por ello, no siempre reproducibles.

\section{Les universidades chinas según la clasificación de Shanghái}

Cuando en 2003 se creó la clasificación de Shanghái, había ocho universidades chinas entre las 500 primeras del mundo, seis entre las 400 y ninguna antes de las 200 (cuadro 1). Comparativamente, España, con menos universidades que China, estaba mejor posicionada globalmente. Una universidad española (Universidad de Barcelona) aparecía entre las 200 mejores y otras 13 entre las 500 mejores. La lista de 2003 la encabezaba la Universidad de Harvard y las 50 primeras posiciones estaban ocupadas principalmente por universidades de Estados Unidos y el Reino Unido. 


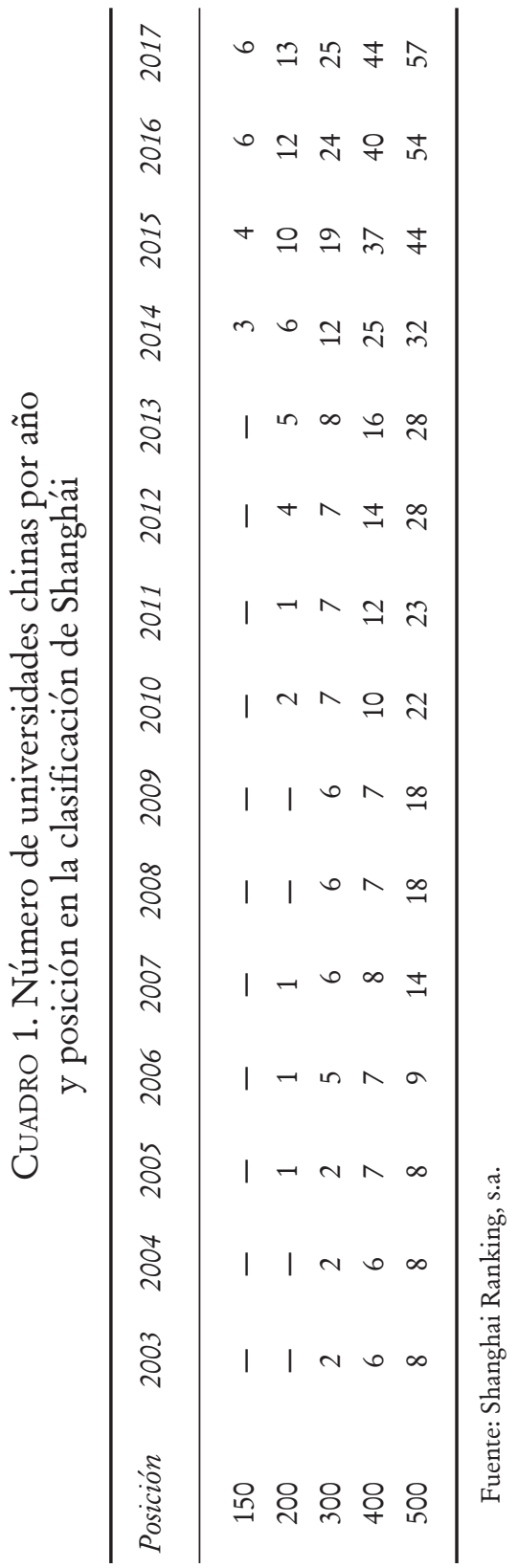


Hasta el año 2005 no apareció la Universidad de Tsinghua entre las 200 primeras posiciones. El número de universidades chinas entre las 500 se mantenía en ocho (cuadro 1). En 2008, la Universidad de Tsinghua descendió de grupo y pasó a estar entre las 300 primeras. El total de las universidades chinas en el top 500 había aumentado a 18. Se iniciaba un proceso de mejora nacional.

Cuando en 2009 se formó la Liga C9 para compartir recursos, personal y resultados, las estadísticas seguían como en 2008 ( 6 en el top 300, 18 en el top 500). En 2010 ya se notó la mejoría tanto local como global. Dos universidades chinas ascendían a las 200 mejores posiciones (Tsinghua y Pekín), siete se encontraban entre las 300 mejores (las anteriores más las de Fudan, Nanjing, Shanghái Jiao Tong, Ciencia y Tecnología de China, y Zhenjiang) y el número en el top 500 se incrementaba a 22 (cuadro 1).

La clasificación de las universidades chinas siguió mejorando hasta que, en 2014, tres universidades se posicionaron entre las 150 primeras (Tsinghua, Pekín y Shanghái Jiao Tong), seis entre las 200 (las tres anteriores más las universidades de Fudan, Ciencia y Tecnología de China, y Zhejiang) y 32 en el top 500. Las otras tres que forman parte de la Liga C9 (Instituto de Tecnología de Harbin y las universidades de Nanjing y de Xi'an Jiaotong), junto con la Normal de Pekín, la de Ciencia y Tecnología de Huazhong y la Sut Yan-Sen, se añadían al grupo top 300. Por lo tanto, todas las universidades que componían la Liga C9 estaban incluidas en el top 300 mundial.

$\mathrm{Al}$ analizar la evolución de cada una de las integrantes de la Liga C9 a lo largo del periodo 2003-2017 (cuadro 2), se observa que la mejoría ha sido progresiva en la mayor parte de los casos.

Sin embargo, aunque China es uno de los países con más producción científica, las listas de la clasificación de Shanghái siguen encabezadas por universidades de Estados Unidos y el Reino Unido. Según el Scimago Journal and Country Rank (s.a.), en el periodo 1996-2016 China publicó 4525851 artículos, lo que la convirtió en el segundo país del mundo con más publicaciones científicas. Estados Unidos ocupó el primer lugar, con 9165271 artículos, y el Reino Unido el tercero, con 


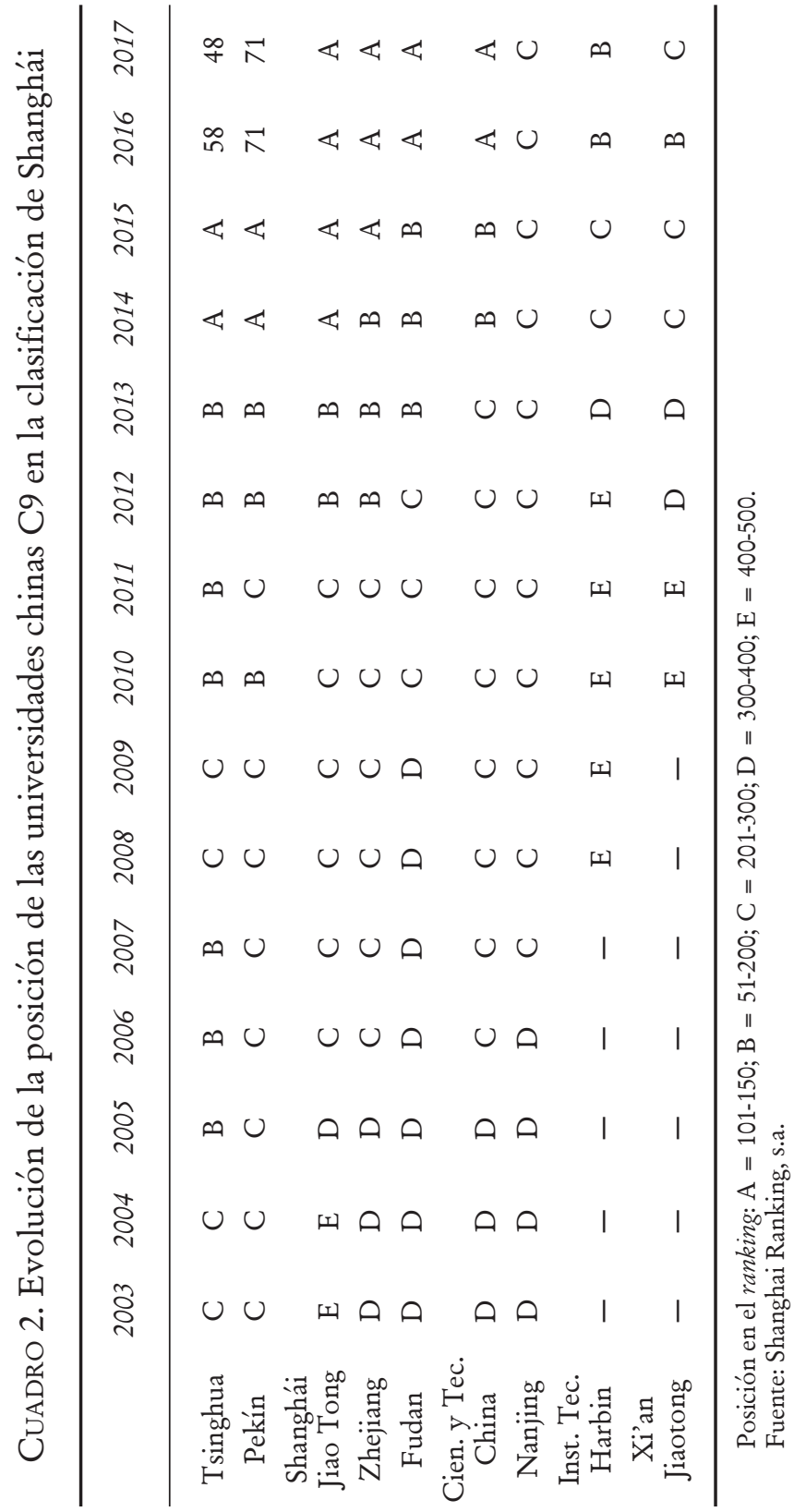


2499 445. Sin embargo, el total de publicaciones sólo refleja la cantidad, no la calidad.

Uno de los factores indicativos de la calidad es el número de veces que los artículos son citados en otras publicaciones. Si se presta atención a este dato, las publicaciones norteamericanas son citadas una media de 23.58 veces por artículo; las del Reino Unido, 21.04, y las de China, 7.16. El conjunto de los datos evidencia el crecimiento cuantitativo de China en innovación, pero no necesariamente en calidad. Además, como se ha señalado, la clasificación de Shanghái tiene en cuenta los artículos publicados en Nature y Science y en revistas indexadas en Thomson Scientific. Es decir, sólo considera textos de muy elevada calidad que, a su vez, suelen ser los más citados.

En el caso de las patentes también se observó un aumento considerable entre 1991 y 2013 (Chen, Patton y Kenney, 2016, p. 916). En 1999, las instituciones chinas de educación superior solicitaron a su país 988 patentes relacionadas con la transferencia de tecnología, mientras que en 2013 hubo 98509 solicitudes. Esto supone una tasa de crecimiento anual de 39\%. Las patentes concedidas por China fueron de 425 en 1999 a 33309 en 2013, una tasa de crecimiento anual de $37 \%$. En 2012, en cambio, las universidades de Estados Unidos recibieron 4797 patentes de su país. Si las oficinas de patentes de ambas naciones concedieran patentes de igual calidad, el sistema universitario chino sería seis veces más productivo que el de Estados Unidos. Sin embargo, si se investigan las licencias derivadas de las patentes chinas (vendidas a empresas y copatentes entre universidades y empresas) se observa que, aunque su número ha aumentado notoriamente, el crecimiento de las licencias ha sido mucho más lento. Esto puede ser resultado de la gran cantidad de incentivos para acrecentar el número de patentes chinas, pero, en la práctica, las que se producen son económicamente menos valiosas.

Aun conociendo estos datos, el gobierno ha fijado el año de 2020 para cuadruplicar la producción de patentes. No obstante, esta orientación cuantitativa no va acompañada de una exigencia explícita de calidad (Fisch, Block y Sandner, 2016, p. 79). Además, las universidades presionan a los estudiantes para producir patentes con el argumento de que será positivo para 
su futuro profesional, lo que provoca una divergencia creciente entre cantidad y calidad (Zhang, Zhao y Lei, 2012, p. 266).

Para que las universidades chinas se aproximen al nivel de las anglosajonas, no sólo es cuestión de aumentar la cantidad de artículos científicos y de sus patentes, sino también de mejorar la calidad (índices de impacto mayor y más licencias de patentes). Alcanzar las primeras posiciones mundiales es una tarea difícil que requiere tiempo y una buena estrategia de investigación, así como financiamiento adecuado. Se requiere, asimismo, una estricta regulación de los gastos realizados para que los fondos se destinen a lo que realmente es necesario.

La corrupción académica que emplea recursos de investigación para viajes injustificados o para comprar equipos caros que no se utilizan es una práctica indeseable que debe ser regulada (Yang y Welch, 2012, p. 647). De hecho, en China la corrupción es una de las grandes preocupaciones en todos los niveles, y el mundo académico no está exento de responsabilidad por posibles malas prácticas. Son tres los aspectos de la educación superior que se ven afectados por este problema: la administración de la investigación, la promoción del profesorado y la formación de los estudiantes de doctorado. La corrupción en la investigación va más allá del plagio y se centra en comportamientos individuales relacionados con la manera en que actúa el propio sistema. Además, mientras que la corrupción económica y política capta mucha atención, la académica es más discreta y suele ser más difícil de detectar (Yang, 2005, pp. 19-20).

En definitiva, mejorar la calidad de la investigación y evitar la corrupción puede suponer, en un futuro no muy lejano, ascender más en la clasificación de Shanghái.

Sin embargo, algunas voces son críticas con el excesivo énfasis que se pone en los rankings y se manifiestan en el sentido de que los perjudicados son los estudiantes. Yu Zhixiang, investigador en química orgánica de la Universidad de Pekín, dice:

El acento en las clasificaciones [ratings] ha afectado negativamente a las facultades. Las universidades gastan mucho dinero en atraer a uno o dos investigadores destacados que dedican todo su tiempo a publicar en revistas académicas indizadas. Los que ejercen la docencia a menudo son los que no han obtenido tan buenos resultados. Para los estudiantes, está lejos de ser lo ideal (Winterbottom, 2013). 
No obstante, los rankings se utilizan con mucha frecuencia y los gobiernos siguen incentivando a las universidades que ocupan las primeras posiciones. Incluso los propios sistemas de elaboración de rankings exploran nuevas clasificaciones que permitan analizar más parámetros para comparar escuelas.

Por ejemplo, desde 2010, además del ranking internacional, la clasificación de Shanghái publica un listado detallado de toda China. En este ranking nacional se cubren cuatro regiones: China continental, Taiwán, Hong Kong y Macao. Aunque los sistemas políticos, económicos y educativos son diferentes, en 2010 Taiwán permitió el acceso a sus universidades a estudiantes de China continental, y actualmente los estudiantes de las cuatro regiones tienen oportunidad de asistir a las universidades de todas las regiones del país. Además, las iniciativas de financiación para conseguir universidades competitivas a nivel internacional también se habían llevado a cabo en Taiwán y Hong Kong, por lo que la comparación en un ranking nacional podía ser interesante.

Para formar parte del ranking nacional, las universidades debían tener o aspirar a una reputación internacional y adquirir el compromiso de aceptar estudiantes de otras jurisdicciones de China. Así, en China continental, entre las 117 universidades del Proyecto 211, donde se incluían las 39 universidades del Proyecto 985, 86 estaban dispuestas a recibir estudiantes extranjeros, de Hong Kong, Macao y Taiwán. Por lo tanto, se consideraron 86 universidades para la clasificación. El mismo procedimiento se aplicó en las otras regiones y se seleccionaron 37 universidades de Taiwán, ocho de Hong Kong y dos de Macao. Finalmente, anualmente se publica un listado de 100 universidades nacionales.

Debido a las diferencias entre los sistemas educativos de las cuatro regiones y las condiciones que se imponían a las universidades para ser consideradas en este ranking nacional, se creyó conveniente modificar algunos indicadores del ranking internacional. Se definieron cuatro categorías: educación, investigación, profesorado y recursos.

- Educación (35\%)

- Porcentaje de estudiantes graduados: 5\% 
- Porcentaje de alumnos no locales: $5 \%$

- Ratio personal académico/estudiantes: $5 \%$

- Número de doctorados: $10 \%$

- Alumnos premio Nobel y con premios destacados: 10\%

- Investigación (35\%)

- Ingreso anual de investigación: 5\%

- Artículos en Nature y Science: 10\%

- Artículos indexados en SCIE y SSCI: $10 \%$

- Patentes internacionales: $10 \%$

- Profesorado (25\%)

- Personal académico con doctorado: $5 \%$

- Personal premios Nobel y premios destacados: 10\%

- Investigadores altamente citados: $10 \%$

- Recursos (5\%)

- Presupuesto anual: 5\%

El proceso de evaluación de las universidades nacionales es el mismo que el de las internacionales. Para cada indicador se asignan 100 puntos a la institución con una valoración más alta y las otras instituciones se calculan como un porcentaje de esta puntuación. En el cuadro 3 se muestran las puntuaciones obtenidas a lo largo de siete años de evaluación de 10 universidades de un total de 100 publicadas. Para elaborar este cuadro, se escogieron las 10 primeras clasificadas en 2014 y se indica entre paréntesis el orden relativo de 2014 y el de los años anteriores y posteriores.

Se observa que casi todas las universidades que aparecen en el cuadro 3 ocupan alguno de los 10 primeros puestos a lo largo de los últimos siete años. Sólo se detectan pequeñas variaciones en las últimas posiciones. También es destacable que las universidades de Hong Kong y Taiwán compiten por las primeras posiciones con las universidades más prestigiosas de China continental (la de Tsinghua y la de Pekín). De hecho, la Universidad Nacional de Tsinghua (Taiwán) se encuentra entre las 150 primeras universidades de la clasificación internacional de Shanghái, por lo que es una competidora directa de las 


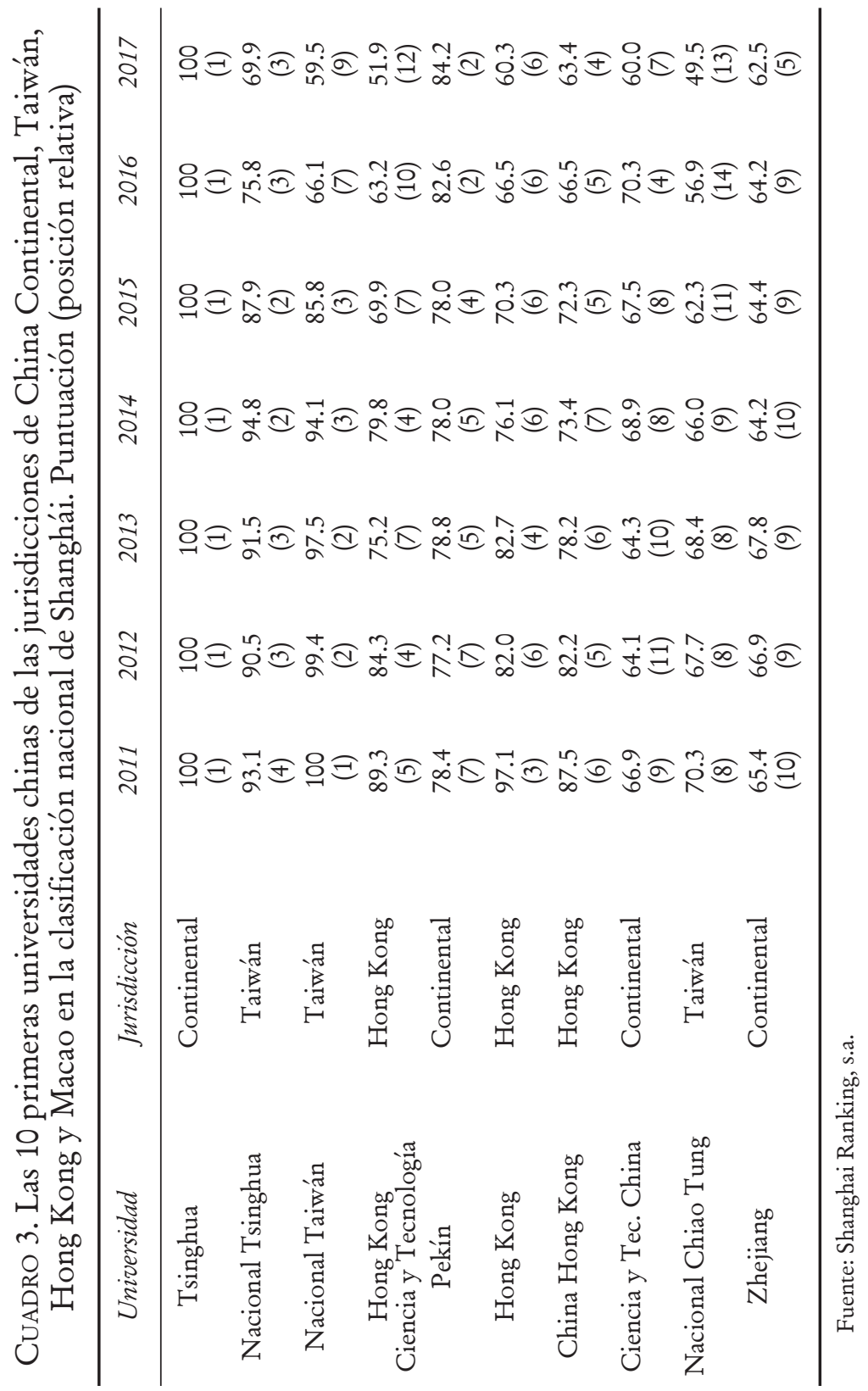


de Tsinghua (continental) y Pekín. La Universidad China de Hong Kong y la de Hong Kong se encuentran entre las 200 primeras, es decir, para efectos del ranking internacional están a la altura de la universidad de Zheijian, la de Fudan y la de Ciencia y Tecnología de China.

Así pues, las nuevas listas nacionales proporcionadas por la clasificación de Shanghái dan una visión interna de la calidad de las universidades chinas, pero no se limitan a las de China continental, sino que se amplían a nuevas jurisdicciones. De alguna forma, estas escuelas también forman parte del mapa universitario chino y, además, son de alta calidad. Por lo tanto, las fronteras se diluyen y la competencia por la excelencia internacional aumenta.

\section{Las universidades chinas de la Liga C9 según otros rankings internacionales}

Las otras dos clasificaciones que más se utilizan, junto con la de Shanghái, son el QS World University Rankings y el Times Higher Education.

Las universidades no suelen hablar de un solo ranking, sino que se fijan en la calificación obtenida en diferentes listados para analizar los aspectos que se pueden mejorar. Es decir, como los indicadores y los sistemas de cálculo de cada ranking son diferentes, las posiciones relativas de cada universidad varían según el registro que se consulte. Aun así, globalmente los resultados de diferentes clasificaciones ofrecen algunos paralelismos y es en el detalle donde se observan diferencias (Khosrowjerdi y Kashani, 2013, p. 2).

Por ejemplo, el QS World University Rankings utiliza seis indicadores: reputación académica (40\%), reputación del ocupador (10\%), ratio estudiantes/profesores (20\%), número de citas científicas (20\%), número de profesores internacionales $(5 \%)$ y número de estudiantes internacionales (5\%). Los valores de los dos primeros indicadores se obtienen a través de encuestas a profesores y ocupadores. El resto se extrae de las estadísticas de las universidades y de la información sobre citas en revistas indexadas que publica Scopus, una de las bases de datos sobre investigación más importantes del mundo. 
Para elaborar el listado QS World University Rankings, se consideran 800 universidades destacadas por su investigación, docencia, ocupabilidad y proyección internacional. Se publican en el ranking los nombres de 500 universidades. Las primeras 400 se clasifican individualmente, y las últimas 100 se colocan en un único grupo. El objetivo de este catálogo es ayudar a los estudiantes a comparar las opciones internacionales, por lo que se incluyen en la evaluación aspectos más docentes, además de los habituales de investigación.

Las posiciones de las universidades que componen la Liga C9 en el QS World University Rankings se presentan en el cuadro 4 en orden descendente a partir del mejor resultado obtenido en 2016.

En general, las posiciones que ocupan las universidades de la Liga C9 en el QS World University Rankings son mejores que las obtenidas en la clasificación de Shanghái. Aun así, las universidades de Estados Unidos y el Reino Unido siguen encabezando las listas. Al comparar los cuadros 3 y 4, se observa que, a grandes rasgos, el orden de las universidades chinas de la Liga C9 es parecido en los dos rankings (Shanghái y QS), a excepción de la Universidad de Fudan, que en 2014 superaba a la Universidad de Shanghái Jiao Tong.

No obstante, como ya se ha comentado, el uso de diferentes indicadores afecta los resultados de evaluación. Por ejemplo, en 2004, la Universidad de Pekín estaba muy bien posicionada en el QS World University Rankings (lugar 17 de 500), pero, aun formando parte de la Liga C9, descendió a partir de 2007 y llegó a ocupar el puesto 57 en 2015 (cuadro 4). De ello se deduce que la investigación que prioriza el Proyecto 985, así como los objetivos de la Liga C9, centrados en optimizar recursos para obtener mejores resultados en investigación, influyen positivamente en la evolución de las posiciones en la clasificación de Shanghái, pero no las favorece necesariamente en el QS World University Rankings.

Esta diferencia podría interpretarse como una disminución de la percepción de la calidad docente de la Universidad de Pekín, ya que los indicadores que se consideran en la clasificación de Shanghái tienen que ver básicamente con la actividad investigadora, pero los del QS World University Rankings 


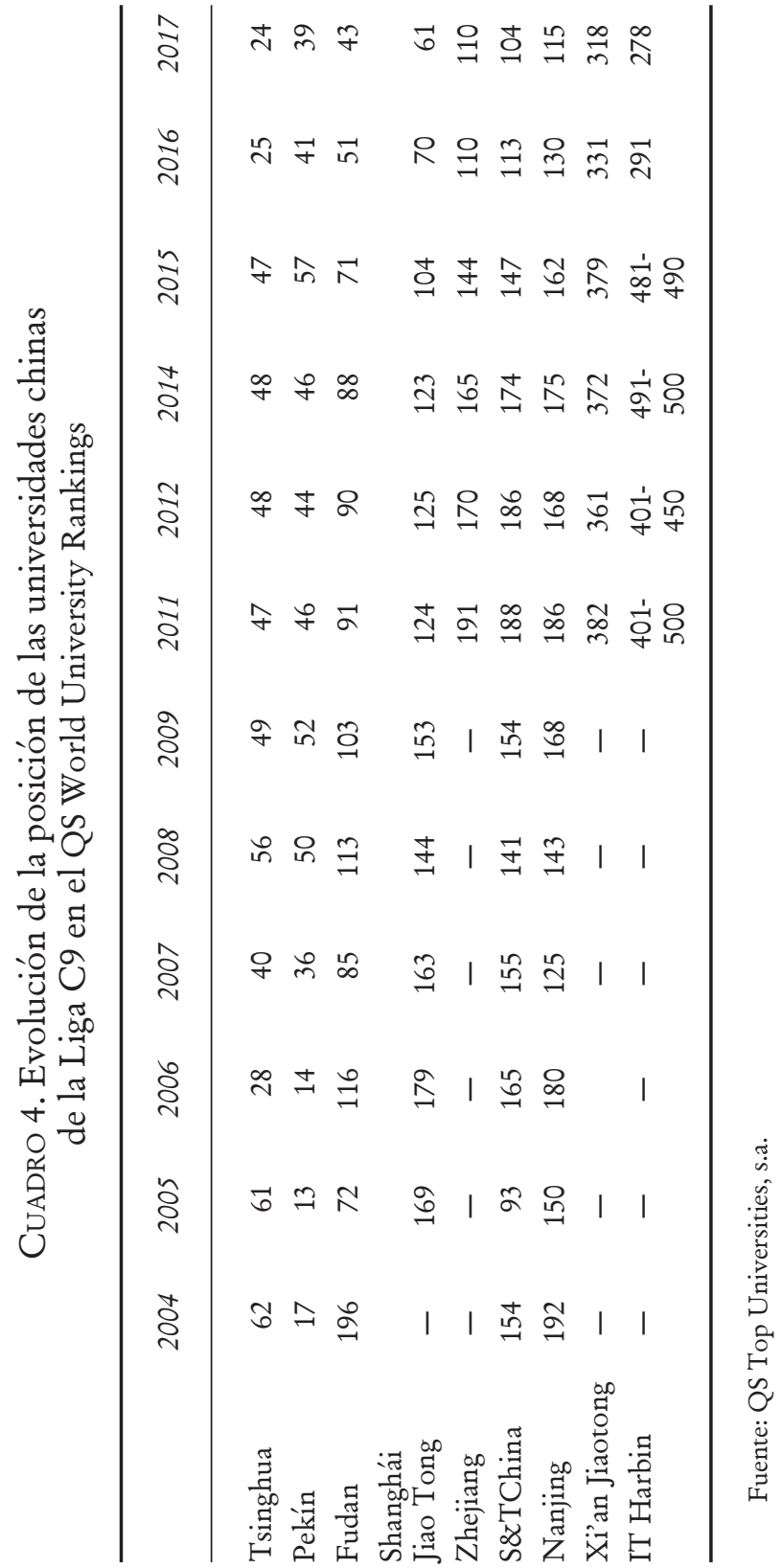


también contemplan la satisfacción y la opinión de los profesores y los empleadores.

En el QS World University Rankings, se identifican las mejores instituciones de cada especialidad (a nivel docente y de investigación) por medio de encuestas a profesores. Puesto que todas las áreas de conocimiento se ven reflejadas en estas encuestas, el indicador de las citas en las publicaciones se equilibra. Mediante las encuestas a los empleadores se identifican las universidades con los mejores graduados e, indirectamente, las que tienen una docencia de mayor calidad. Así pues, la incorporación de nuevos datos referidos a más aspectos docentes puede cambiar la evolución de las universidades con el tiempo e incluso alterar el orden puntual de la clasificación, como se ha visto en el caso de la Universidad de Pekín.

En el segundo ejemplo, el Times Higher Education utiliza 13 indicadores agrupados en cinco áreas: docencia (calidad del entorno de aprendizaje, 30\%), investigación (volumen, ingresos y reputación, 30\%), citas (influencia de las investigaciones, $30 \%$ ), ingresos de la industria (aspectos de innovación, 2.5\%) e internacionalización (personal, estudiantes e investigación, 7.5\%). El listado de las 400 mejores universidades se publica anualmente desde 2010-2011. En este ranking se excluyen las universidades que no ofrecen estudios de grado, las que se dedican sólo a un área de conocimiento y las que publicaron menos de 200 artículos por año entre 2008 y 2012.

De la misma forma que en el QS World University Rankings, la evaluación de la calidad de la docencia y de parte de la investigación en el Times Higher Education se realiza a partir de encuestas a profesores y de datos docentes, pero el peso de la producción científica es superior en el caso de este último. Por lo tanto, es de esperar que el orden de las universidades también varíe respecto a la clasificación de Shanghái (cuadro 5).

Según el Times Higher Education, Pekín y Tsinghua siguen siendo las universidades chinas mejor valoradas, con una distancia considerable del resto. En general, las otras universidades de la Liga C9 se han mantenido estables o han fluctuado ligeramente desde 2011, excepto la Universidad de Fudan, que ha experimentado una mejoría progresiva hasta situarse en el lugar 155 del ranking, inferior todavía al de las dos universi- 


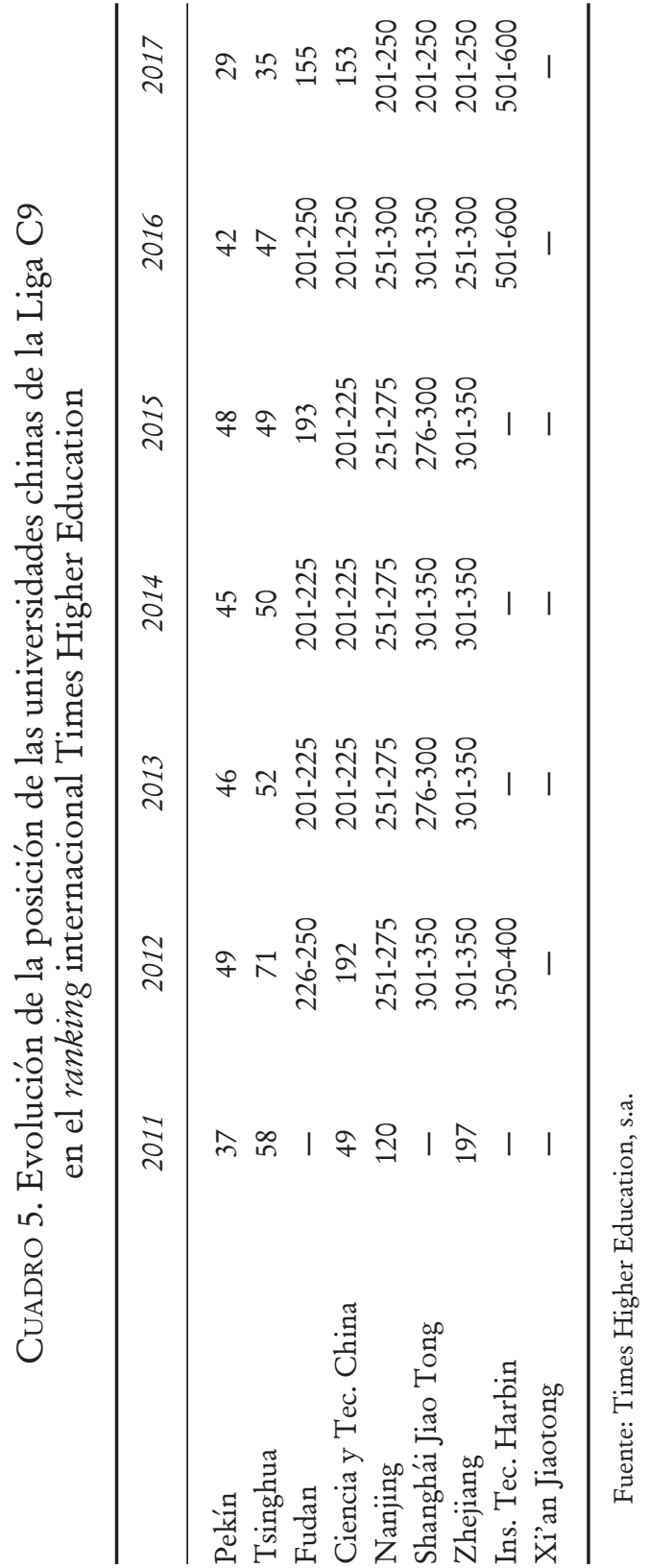


dades líderes. La ordenación relativa y la comparación con la clasificación de Shanghái conducen a conclusiones parecidas a las formuladas para el QS World University Rankings.

En definitiva, la evolución general de las universidades chinas en diferentes rankings demuestra que las estrategias aplicadas por los Proyectos 211 y 985 sobre el grupo de las instituciones de la Liga C9 han dado buenos resultados. Desde la aplicación de los planes de investigación de los gobiernos de Jiang Zemin y $\mathrm{Hu}$ Jintao se han visto potenciadas las universidades chinas de élite. En este sentido, en marzo de 2015, Wang Huiyai, director del Center for China and Globalization, atribuía los buenos resultados generales al apoyo del gobierno, así como a los esfuerzos realizados por las propias universidades. A su vez, Jiang Guohua, director del Comité Académico de la página cuua.net (Chinese Universities Alumnus Association) iba más allá y afirmaba:

En lugar de decir que el mundo está valorando mejor las dos universidades chinas [Pekín y Tsinghua], preferiría decir que ve con optimismo el futuro de la economía china. En este contexto, la gente cree que la Universidad de Pekín y la Universidad de Tsinghua jugarán un papel más crítico en el futuro (Zhao, 2015).

Sin embargo, los datos que se extraen de los rankings se pueden interpretar de muchas maneras y a diferentes niveles. Es importante conocer los indicadores utilizados en su elaboración para modificar estrategias concretas, que deben potenciar proyectos innovadores, originales y que abran nuevas vías de investigación que resulten de interés para los grupos más excelentes. Sólo así aumentará el número de citas en las revistas indexadas y en las publicaciones de más alto nivel, factores esenciales para mejorar las posiciones de las universidades de la Liga C9 en los rankings analizados.

\section{Conclusiones}

El objetivo principal de los Proyectos 211 y 985 era que las universidades chinas tuvieran un grado de excelencia internacional, especialmente en investigación. Por este motivo, se financió 
con más presupuesto a ciertas universidades escogidas por el gobierno. Nueve de ellas conformaron la Liga C9 y recibieron un presupuesto extraordinario.

Según la clasificación de Shanghái, las universidades de la Liga C9 han experimentado una gran mejora en los últimos 10 años, y tres de ellas (Tsinghua, Pekín y Shanghái Jiao Tong) se encuentran entre las 150 mejores del mundo. Las dos primeras (Pekín y Tsinghua) también tienen los mejores resultados en el QS World University Rankings y el Times Higher Education.

El resto de las universidades que conforma la Liga C9 se encuentran entre las mejores 300 del mundo según la clasificación de Shanghái y, si se tiene en cuenta que partían de posiciones mucho más desfavorables, su ascenso resulta espectacular.

Cabe destacar también que Taiwán, Hong Kong y Macao han acordado con China continental la apertura de las fronteras académicas para que los estudiantes de las cuatro jurisdicciones puedan estudiar donde lo deseen. Esto pone a más universidades en el mapa de los rankings. De hecho, según los resultados del ranking mundial, tres universidades de Hong Kong y tres de Taiwán compiten fuertemente con las mejores universidades de la Liga C9 de China continental.

Aun así, la lista sigue encabezada mayoritariamente por universidades de Estados Unidos y el Reino Unido. Romper el dominio anglosajón implica diferentes retos para las universidades chinas. El más importante es apostar por una mejora de la calidad de la investigación. Hasta el momento, la estrategia del gobierno ha sido invertir recursos para aumentar la producción científica. Las estadísticas demuestran que lo han conseguido, porque el número de artículos y de patentes ha aumentado enormemente en los últimos años. Sin embargo, aunque se haya incrementado la cantidad, parece que la calidad es mejorable. En esta fase es necesario un cambio de orientación en las políticas que deciden la financiación de los grupos de investigación, y, para evitar la corrupción académica, un presupuesto dirigido a proyectos más innovadores y que hagan crecer el interés internacional.

Las universidades chinas tienen, pues, un importante reto en el futuro cercano. Si el gobierno sigue ubicando la ciencia y 
la tecnología como objetivos de primer orden para el desarrollo del país, debe aportar un presupuesto considerable e incentivar la calidad de la producción científica. Actualmente, las universidades chinas son competitivas internacionalmente, pero podrían serlo más.

Dirección institucional de la autora:

Universidad Rovira i Virgili

Campus Sescelades

C/Marcellí Domingo, $s / n$

43007 Tarragona, España

\section{Referencias}

Billaut, J.-C., Bouyssou, D. y Vincke, P. (2010). Should you believe in the Shanghai ranking? Scientometrics, 84(1), 237-263. https:// dx.doi.org/10.1007/s11192-009-0115-x

Chen, A., Patton, D. y Kenney, M. (2016). University technology transfer in China: A literature review and taxonomy. Journal of Technology Transfer, 41(5), 891-929. https://dx.doi.org/10.1007/ s10961-016-9487-2

Docampo, D. y Cram, L. (2014). On the internal dynamics of the Shanghai ranking. Scientometrics, 98(2), 1347-1366. https://dx. doi.org/10.1007/s11192-013-1143-0

Fisch, C. O., Block, J. H. y Sandner, P. G. (2016). Chinese university patents: Quantity, quality, and the role of subsidy programs. The Journal of Technology Transfer, 41(1), 60-84. https://dx.doi. org/10.1007/s10961-014-9383-6

Flórez-Parra, J. M., López-Pérez, M. V. y López-Hernández, A. M. (2014). El gobierno corporativo de las universidades: estudio de las cien primeras universidades del ranking de Shanghái. Revista de Educación, (364), 170-196. Recuperado de https://sede. educacion.gob.es/publiventa/d/16365/19/0

Heron, L. (15 de octubre de 2013). Nine Chinese universities sign academic freedom pact. South China Morning Post. Recuperado de http://www.scmp.com/news/china/article/1331803/ninechinese-universities-sign-academic-freedom-pact

KhosRowjerdi, M. y Kashani, Z. S. (2013). Asian top universities in six world university ranking systems. Webology, 10(2), 1-9. Recuperado de http://www.webology.org/2013/v10n2/a114.pdf 
National Bureau of Statistics of China. (2013). China Statistical Year Book 2013. Recuperado de http://www.stats.gov.cn/tjsj/ ndsj/2013/indexeh.htm

QS Top Universities. (s.a.). University Rankings. Recuperado de http://www.topuniversities.com/university-rankings

Scimago Journal and Country Rank. (s.a.). International Science Ranking. Recuperado de http://www.scimagojr.com/countryrank.php

Shanghai Ranking. (s.a.). Academic Ranking of World Universities. Recuperado de http://www.shanghairanking.com/

Times Higher Education. (s.a.). World University Rankings. Recuperado de https://www.timeshighereducation.com/world-university-rankings

WinterbotTOM, V. (13 de julio de 2013). Improving rankings for Chinese universities. China Outlook. Focusing on China's Future. Recuperado de http://chinaoutlook.com/improving-rankingsfor-chinese-universities/

XiAo, Z. y Dyson, J. R. (1999). Chinese students' perceptions of good accounting teaching. Accounting Education, 8(4), 341-361. https://dx.doi.org/10.1080/096392899330838

YANG, R. (2005). Corruption in China's higher education system: A malignant tumor. International Higher Education, (39), 18-20. Recuperado de https://ejournals.bc.edu/ojs/index.php/ihe/ article/view/7473/6668

YANG, R. y Welch, A. (2012). A world-class university in China? The case of Tsinghua. Higher Education, 63(5), 645-666. http:// dx.doi.org/10.1007/s10734-011-9465-4

Zhang, G., Zhao, Y. y Lei, J. (2012). Between a rock and a hard place: Higher education reform and innovation in China. On the Horizon, 20(4), 263-273. http://dx.doi.org/10.1108/ 10748121211272489

Zhao, X. (31 de marzo de 2015). Tsinghua, Peking universities rise in global ranking. China Daily. Recuperado de http://www. chinadaily.com.cn/china/2015-03/31/content_19958578.htm

Zhao, L. y Zhu, J. (2010). China's higher education reform: What has not been changed? East Asian Policy, 2(4), 115-125. 
